\title{
Adaptive Sensor Data Fusion in Motion Capture
}

\author{
Shuyan Sun, Xiaoli Meng, Lianying Ji and Jiankang Wu \\ Graduate University of Chinese Academy of Sciences \\ Beijing, China \\ China-Singapore Institute of Digital Media \\ Singapore \\ sunshuyan@csidm.sg, mengxiaoli07@mails.gucas.ac.cn, \\ jilianying@guacas.ac.cn, jkwu@gucas.ac.cn
}

\author{
Wai-Choong Wong \\ Department of Electrical and Computer \\ Engineering \\ National University of Singapore \\ Singapore \\ wong_lawrence@nus.edu.sg
}

\begin{abstract}
Micro-sensor human motion capture has shown its potentials because of its ubiquity and low cost. One of the biggest challenges in micro-sensor motion estimation is the drift problem caused by integration of angular rates to obtain orientation. To reduce the drift, existing algorithms make use of gravity and earth magnetic filed measured by accelerometers and magnetometers respectively. Unfortunately, body segment acceleration and environment magnetic disturbance produce strong interferences to the gravity and earth magnetic field measurement respectively. This paper presents a novel sensor fusion algorithm for driftfree orientation estimation, where a quaternion-based complementary Kalman filter is designed. To optimize the performance under interference, this filter fuses gyroscope, accelerometer and magnetometer signals adaptively based on their information confidence, which are evaluated by computing their interference level. The proposed algorithm showed least error compared with the existing methods in the quantitative experiments, and its effectiveness was also verified by the stable and accurate human motion estimation.
\end{abstract}

Keywords: Sensor fusion, adaptive weighting, Kalman filtering, human motion capture, drift.

\section{Introduction}

Human motion capture (Mocap) has wide applications in many areas, such as virtual reality, interactive game and learning, animation and film special effects, etc. Among all the motion capture techniques, optical motion capture is one of the most mature techniques, which is widely used by the film industry, producing successful movies, such as "Lord of the Rings" and "Avatar". However, an optical human motion capture system has certain limitations. First of all, there is a need for multiple high speed and high resolution cameras structured and calibrated in the dedicated studio, which restricts applications into a laboratory-like environment. Secondly, the system is quite complex and there is a huge amount of data to be processed. Moreover, optical motion capture system is usually very expensive and inconvenient to most applications.
With the rapid advances of micro-electro-mechanical systems (MEMS) sensors, the research on human motion capture using micro-inertial sensors becomes more attractive. In Micro-sensor Motion capture (MMocap), micro inertial / magnetic sensors are attached to body segments. Segment orientation can be estimated from the fusion of sensory data. Based on the estimated orientation, together with the length of each segment and the arranging relationship between segments, the motion of the whole body can be obtained. MMocap has no line-ofsight requirements, and no emitters to install. Thus, MMocap systems can be applied in a variety of applications where laboratory-like environment is not necessary. The motion measurements in MMocap are relatively direct, and the system is relatively simple, at least not as complicated as optical Mocap systems. Moreover, a MMocap system costs much lower than that based on the optical Mocap.

However, there are technical challenges due to its inherit characteristics in MMocap. From the data fusion point of view, there are two levels of fusion among these challenges: the first level is the fusion of three types of micro sensors contained in a micro Sensor Measurement Unit (SMU), namely, 3D micro-gyroscope, 3D accelerometer and 3D magnetometer. The second level is fusion of motion estimation of individual body segments to form the whole body motion. This paper discusses the first level of data fusion to reduce the drift of orientation estimation. In MMocap there is a SMU node attached to every body segment. The gyroscope is used as a main data source, which measures angular rates, by the integration of which orientation of that body segment can be obtained. As the result of integration, the errors also accumulate. This results in a drift over a period of time. Because the drift of each body segment is most likely toward a different direction, leading to inconsistency issues at the second level information fusion. In order to reduce the drift in the data fusion algorithms, the accelerometer is used to measure the gravity vector relative to the sensor coordinate system, and allows accurate determination of pitch and roll. The magnetometer, based on the principle of a compass to measure the earth magnetic field, is used to measure azimuth or rotations about the vertical axis. The data from these three kinds of sensors are usually 
fused in an algorithm to "eliminate" the drift and to derive the orientation estimation.

There are many fusion algorithms to obtain orientation from gyroscope, accelerometer and magnetometer signals. Foxlin et al. [1] proposed a complementary separate-bias Kalman filter, which was designed for head tracking applications. Yun et al. [2] presented an extended Kalman filter designed for real-time estimation, where a preprocessing of the acceleration and magnetometer measurements was implemented to simplify the complexity of the filter. Recently they proposed a factored quaternion algorithm (FQA) [3], which restricts the use of magnetic data to the determination of the azimuth angle. They illustrated that FQA was computationally more efficient and the magnetic variations caused only azimuth errors in attitude estimation. Roetenberg et al. [4] described a complementary Kalman filter design, which compensated the magnetic disturbance by estimating the disturbance error and adaptively changing the measurement covariance based on the disturbance estimation. Kraft [5] described an unscented quaternionbased Kalman filter for real-time estimation of a rigid body orientation. Simulation results demonstrate the general validity of the described filter. Among all the studies mentioned above, both Foxin [1] and Roetenberg [4] fused inertial and magnetic sensing under the framework of the complementary filter, which is suitable for combining complementary data sources. Kraft [5] and Yun [2,3] expressed orientation by quaternions, which do not suffer from the singularity problem. The FQA proposed by Yun [3] restricted the use of the magnetometer, and the inference from magnetic disturbance to roll and pitch was removed.

Considering the state-of-the-art of MMcap, we propose a novel data fusion algorithm for motion estimation using micro SMU's. The algorithm performs cross modality information fusion to estimate orientation with very low drift. One of the contributions of this paper is the complementary Kalman filter (CKF), which is quaternionbased. From gyroscope signals, orientation can be integrated from angular rates, which has reasonable accuracy within a short time. From the gravity component of accelerometer signals, pitch and roll angle can be obtained accurately, but there will be problems if the body acceleration can not be neglected with comparison to gravity. From magnetometer signals, azimuth angle can be obtained accurately when there is no magnetic disturbance. All of these three data sources of different modality cannot determine the accurate orientation alone, rather, they are complementary to each other. To make use of all those three available sensor data efficiently, the CKF is designed, which is suitable for combining complementary data sources. In the designed CKF, quaternions are selected to represent the rotation and orientation of each body segment, for they do not suffer from the singularity problem. Another contribution of this paper is the compensation of magnetic disturbance and body segment acceleration, since they are interferences to earth magnetic field and gravity measurement, respectively. This filter fuses accelerometer and magnetometer data adaptively based on their confidence which are evaluated using the level of acceleration and magnetic disturbance. The good performance of the experimental results has shown the feasibility and effectiveness of the proposed algorithm.

The rest of the paper is organized as follows. Section 2 describes the quaternion-based CKF for sensor fusion. Section 3 presents the experimental results. Finally, conclusions and future work are given in Section 4.

\section{Proposed Sensor Fusion Algorithm}

The proposed CKF takes a quaternion to represent the orientation of each body segment. Under the filtering framework, the integrated quaternion from gyroscope signals is corrected by the gravity and earth magnetic direction for drift-free orientation adaptively based on the confidence of each data source. This section first describes the quaternion representation, which is followed by the filter process model and measurement model employed by the CKF. Then the confidence computation and filter design are explained.

\subsection{Quaternion Representation}

In MMocap, a SMU is fixed on a body segment. Before the analysis it is necessary to define the coordinate systems. First, there exists a Global Coordinate System (GCS) which is earth related and time invariant. GCS is taken as the reference frame. Second, a Body Coordinate System (BCS) is attached to the body segment which is time variant coinciding with segment motion. The orientation between body frame and reference frame needs to be determined. The Sensor Coordinate System (SCS) is defined by the SMU itself and also coincides with segment motion. Since the SMU is rigidly attached to the body segment, the orientation between BCS and SCS is a constant offset. For the convenience of analysis, it is assumed that SCS coincides with BCS.

Quaternions are used to represent the rotation and orientation of the body segment, because they do not suffer from the singularity problem, which is associated with Euler angle / rotation matrix representation and is well known as the Gimbal Lock. Moreover, quaternions avoid trigonometric functions in the filtering algorithm, making their computation more efficient compared to rotation matrixes.

Quaternions consist of a vector part $e=\left[q_{1}, q_{2}, q_{3}\right]^{T} \in \boldsymbol{R}^{3}$ and a scalar part $q_{4} \in \boldsymbol{R}[6]$.

$$
q=\left[e^{T}, q_{4}\right]^{T}=\left[\begin{array}{l}
q_{1} \\
q_{2} \\
q_{3} \\
q_{4}
\end{array}\right]
$$

Any given vector $r \in \boldsymbol{R}^{3}$ in the reference frame can be rotated into the body frame by a unit quaternion $q$ 


$$
b=h(q)=C(q) r
$$

where $b \in \boldsymbol{R}^{3}$ is the representation of vector $r$ in the body frame,

$$
\begin{gathered}
C(q)=\left(q_{4}^{2}-e^{T} e\right) I_{3}+2 e e^{T}-2 q[e \times] \\
{[e \times]=\left[\begin{array}{ccc}
0 & -q_{3} & q_{2} \\
q_{3} & 0 & -q_{1} \\
-q_{2} & q_{1} & 0
\end{array}\right]}
\end{gathered}
$$

In what follows, $r$ denotes the true gravity / earth magnetic direction unit vector in the reference frame with $b$ denoting that in the body frame.

It is well-known that quaternions satisfy the following differential equation [6]:

$$
\frac{d}{d t} q=\frac{1}{2} \Omega[\omega] q
$$

where $\Omega[\omega]$ is a $4 \mathrm{x} 4$ skew symmetric matrix

$$
\Omega[\omega]=\left[\begin{array}{cc}
-[\omega \times] & \omega \\
-\omega^{T} & 0
\end{array}\right]
$$

And $\omega$ is the angular velocity

$$
\omega=\left[\begin{array}{l}
\omega_{x} \\
\omega_{y} \\
\omega_{z}
\end{array}\right]
$$

with $[\omega \times]$ representing cross product operator:

$$
[\omega \times]=\left[\begin{array}{ccc}
0 & -\omega_{z} & \omega_{y} \\
\omega_{z} & 0 & -\omega_{x} \\
-\omega_{y} & \omega_{x} & 0
\end{array}\right]
$$

Given the sampling interval $\Delta$, if the angular velocity is constant in the interval $[(t-1) \Delta, t \Delta]$, then equation (5) can be extended into the discrete-time model:

$$
q_{t}=\exp \left(\frac{1}{2} \Omega[\omega] \Delta\right) q_{t-1}
$$

However, this equation only holds when the angular velocity is constant in the interval $[(t-1) \Delta, t \Delta]$.

\subsection{Process Model}

The process model employed by the CKF governs the dynamic relationship between states of two continuous time steps. Instead of quaternion estimate, the estimation error between the true and integrated quaternion is taken as the filter state vector. The process model is derived under the framework of an error state model [4], which is used to represent the relationship between errors in the estimated state variables and the errors in the gravity and magnetic direction unit vectors. In what follows, the system transition matrix and noise model will be derived.

It is assumed that the relationship between the estimation error and the estimated quaternion is a linear function:

$$
q_{t, \varepsilon}=q_{t}-\hat{q}_{t}
$$

where $q_{t, \varepsilon}$ is the estimation error, $q_{t}$ is the true orientation quaternion, the subscript $t$ denotes time and $\hat{q}_{t}$ is the estimation, which is predicted by the integration of the angular rates measured by the gyroscope, as shown in (9).

The gyroscope measures the angular velocity with bias and noise,

$$
y_{G, t}=\omega_{t}+{ }^{G} a_{t}+v_{G, t}
$$

where $v_{G, t}$ is the gyroscope noise modelled as a Gaussian noise, $N\left(0, \Sigma_{G}\right)$, and ${ }^{G} a_{t}$ is the bias vectors (assumed to be null). Here the superscript ' $G$ ' means gyroscope. Substituting (10) and (11) into (9) and taking the angular velocity error into account, we have:

$$
\begin{aligned}
\hat{q}_{t} & =\exp \left(\frac{1}{2} \Omega\left[y_{G, t}\right] \Delta\right) \hat{q}_{t-1} \\
& \cong q_{t}-A_{t} q_{t-1, \varepsilon}+\frac{\Delta}{2} \Xi_{t-1} v_{G, t}
\end{aligned}
$$

where

$$
\begin{aligned}
& A_{t}=\exp \left(\frac{1}{2} \Omega\left[y_{G, t}\right] \Delta\right) \\
& \Xi_{t}=\left[\begin{array}{c}
{\left[e_{t} \times\right]+q_{t, 4} I_{3}} \\
-e_{t}^{T}
\end{array}\right]
\end{aligned}
$$

The approximation in (12a) is obtained in the same way as in [6].

Comparing (12a) with (10), we have

$$
q_{t, \varepsilon} \cong A_{t} q_{t-1, \varepsilon}-\frac{\Delta}{2} \Xi_{t-1} v_{G, t}
$$

Thus, the error-state dynamic equation is given by

$$
\begin{aligned}
q_{t, \varepsilon} & =A_{t} q_{t-1, \varepsilon}+w_{t} \\
w_{t} & =-\frac{T}{2} \Xi_{t-1} v_{G, t}
\end{aligned}
$$

where $w_{t}$ is the system noise modelled as a zero mean Gauss distribution, whose covariance matrix is given by

$$
Q_{t}=\left(\frac{\Delta}{2}\right)^{2} \Xi_{t-1} \Sigma_{G} \Xi_{t-1}^{T}
$$

\subsection{Measurement Model}

The measurement model employed by the CKF governs the relationship between the error state and sensor signals. From sensor signals, we obtain

$$
y_{S, t}=B_{t}+{ }^{s} A_{t}+V_{S, t}
$$

where $V_{S, t}$ is the accelerometer / magnetometer measurement noise, which is modelled as a Gaussian distribution, $N\left(0, \Sigma_{S y}\right) .{ }^{S} A_{t}$ is the bias vector (assumed to be null). Here the superscript ' $S$ ' means accelerometer / magnetometer. $B_{t}$ denotes the gravity plus body acceleration, or the earth magnetic field plus disturbance, which is sampled in the sensor frame. In this section, the 
interference from body acceleration and magnetic disturbance, which will be detailed in the next section, is ignored.

It can be seen that $y_{S, t}$ contains both magnitude and orientation information. The useful information contained in sensor data is the direction of gravity and earth magnetic field, while the magnitude error will reduce the orientation precision without sensor signal normalization. Thus normalization will be performed to (17a) first,

$$
z_{S, t}=b_{t}+{ }^{S} a_{t}+v_{S, t}
$$

where $v_{S, t}$ is the measurement Gaussian noise after normalization, whose covariance is $\Sigma_{s} ;{ }^{S} a_{t}$ is the bias vectors after normalization (also assumed to be null); $b_{t}$ denotes the true gravity / earth magnetic direction vector.

As shown in (2), given a quaternion, $q_{t}$, the gravity / earth magnetic unit direction vector $r$ in reference frame can be rotated into the body frame. Therefore the gyroscope-based estimated gravity / earth magnetic direction can be derived,

$$
\hat{z}_{S, t}=h\left(\hat{q}_{t}\right)
$$

where the estimated quaternion in (18) is predicted from (9) using the gyroscope data. (18) is a non-linear function of the quaternion. Given the gyroscope-based estimate,

$$
\hat{q}_{t}=q_{t}-q_{t, \varepsilon}
$$

The true direction vector in the body frame can be obtained by the vector Taylor series expansion,

$$
\begin{aligned}
b_{t} & =\hat{z}_{S, t}+H_{\hat{q}_{t}}\left(q_{t}-\hat{q}_{t}\right)+H O T \\
& =\hat{z}_{S, t}+H_{\hat{q}_{t}} q_{t, \varepsilon}+H O T
\end{aligned}
$$

where $H_{q}$ is the Jacobian matrix of partial derivatives of $h(q)$ with respect to $q$; HOT stands for higher order terms.

Combining (17b) with (19) and neglecting the HOT yields the measurement function,

$$
z_{t, \varepsilon}=z_{S, t}-\hat{z}_{S, t} \cong H_{\hat{q}_{t}} q_{t, \varepsilon}+v_{S, t}
$$

Note that the quaternion from (9-10) is not a unit quaternion, $H_{q}$ will not be of full rank and the resulting Jacobian matrix will be singular and non-invertible. Constraining the quaternion to be of unit length will eliminate this problem. Thus, before the calculation of $H_{q}$, normalization has to be done as follows,

$$
\bar{q}=\frac{q}{\|q\|}
$$

\subsection{Adaptive Weighting}

Under the CKF framework, the integrated quaternion from the gyroscope signals is corrected by the gravity and earth magnetic direction, but the accuracy will decrease with the introduction of body acceleration and magnetic disturbance contained in accelerometer and magnetometer signals. Thus, the confidence of each data source need to be determined based on which the filter can fuse sensor information adaptively to obtain more accurate orientation.
From the sensor we can obtain the standard gravity / magnetic field measurement, $r_{0}$, which denotes the gravity without sensor motion, or the earth magnetic field without disturbance. Then the magnitude and direction of $r_{0}$ can be obtained,

$$
\begin{gathered}
r_{0, m}=\left\|r_{0}\right\| \\
r_{0, d}=\frac{r_{0}}{r_{0, m}}
\end{gathered}
$$

where the subscripts ' $m$ ' and ' $d$ ' denote magnitude and direction respectively.

Given the standard gravity / magnetic field, $r_{0}$, when the accelerometer / magnetometer signals $y_{S, t}$ are available, the error of the magnitude and direction of these signals are firstly computed. The magnitude and direction of $y_{S, t}$ are represented as $y_{s, t, m}$ and $y_{s, t, d}$. The magnitude error is derived as:

$$
E_{m}=\max \left(\frac{y_{S, t, m}}{r_{0, m}}, \frac{r_{0, m}}{y_{S, t, m}}\right) \in[1,+\infty)
$$

The reason why the maximum operation is taken here is that the magnitude error $E_{m}$ will tend to infinity when $y_{s, t, m}$ tends to both infinity and zero. The direction error is given by:

$$
\begin{aligned}
E_{d} & =\left\|r_{0, d}-q_{t} \otimes y_{S, t, d} \otimes\left(q_{t}\right)^{-1}\right\| \\
& =\left\|C\left(q_{t}\right) r_{0, d}-y_{S, t, d}\right\| \in[0,2]
\end{aligned}
$$

where $\otimes$ denotes quaternion multiplication, $\|\cdot\|$ represents $l^{2}$-norm, and $C$ is the same as in (3). The total error considering both magnitude and direction is governed by

$$
E=E_{d} \cdot E_{m} \in[0,+\infty)
$$

Thus the confidence of the information $y_{S, t}$ is given by

$$
f\left(y_{S, t}\right)=e^{-\alpha_{S} E}
$$

where the coefficient $\alpha_{S}$ is a positive real number and is data-source dependent. The purpose that the exponential distribution is used to represent the confidence of each kind of sensor is to decrease the weighting of the sensor exponentially when there is interference, which makes the filter less dependent on this data source.

Given the original sensor measurement noise covariance $\Sigma_{S}$ when there is no error driven from body acceleration or magnetic disturbance, the covariance which will be put into the Kalman filter is given by

$$
\Sigma_{b}=e^{\alpha_{S} E} \Sigma_{S}
$$

When there is body acceleration / magnetic disturbance, the confidence of the sensor data computed from (27) will exponentially decreased, then the filter will put less weight on this data through (28).

\subsection{Filter design}

The proposed CKF operates on the error state model, while the rotation quaternion estimation error $q_{t, \varepsilon}$ is taken 
as the state vector as given in (10), and the system function is

$$
q_{t, \varepsilon}=A_{t} q_{t-1, \varepsilon}+w_{t}
$$

where $A_{t}$ is the system transition matrix,

$$
A_{t}=\exp \left(\frac{1}{2} \Omega\left[y_{G, t}\right] \Delta\right)
$$

And $w_{t}$ is the system noise which is governed by

$$
w_{t}=-\frac{\Delta}{2} \Xi_{t-1} v_{G, t}
$$

Its covariance matrix is given by (16).

The difference between the gravity / earth magnetic direction unit vector from the inertial prediction and that from accelerometer / magnetometer signals, $z_{t, \varepsilon}$, is used as the measurement vector to update the estimated state,

$$
z_{t, \varepsilon}=z_{S, t}-\hat{z}_{S, t}
$$

where $z_{S, t}$ are the accelerometer / magnetometer signals as defined in (17b). The last term in (32) is the estimated gravity / magnetism unit direction vector derived by the predicted quaternion integrated from gyroscope signals as shown in (18). The measurement function of the filter is given by:

$$
z_{t, \varepsilon}=H_{\hat{q}_{t}} q_{t, \varepsilon}+v_{S, t}
$$

where $v_{S, t}$ is the measurement noise, whose covariance is given by (28), which is related to the information confidence of each data source.

In summary, the work flow of our filtering algorithm is given as follows and is as shown in Fig.1:

a) At time $t$, the gyroscope, accelerometer and magnetometer signals become available.

b) Predict the orientation quaternion using gyroscope information using (9), and normalize the resulting quaternion.

c) Calculate the Kalman filter system transition matrix and system noise covariance through (30) and (16) respectively.

d) Calculate the Jacobian matrix of the measurement function, error measurement input and the measurement noise covariance through (33), (32) and (24-28) respectively.

e) Set the estimated error state input as a zero vector, and do Kalman filtering.

The estimated quaternion error from filtering will be combined with the predicted quaternion from gyroscope integration to obtain the final quaternion estimation, which will be used for the next iteration. The standard Kalman filter process can be found in many textbooks, which is not detailed here.

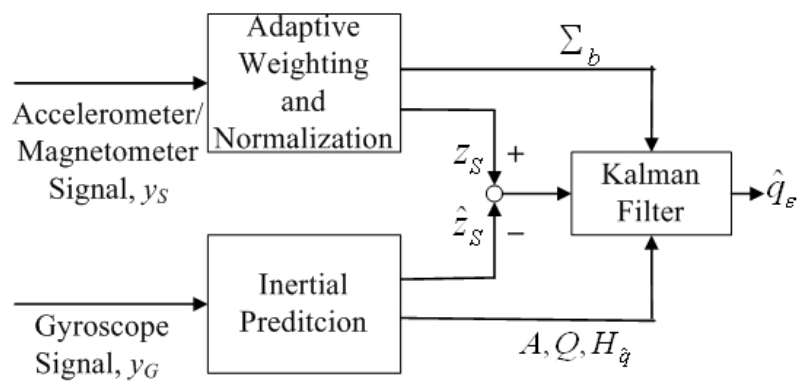

Fig.1 Work flow of the CKF

\section{Experimental Results}

For the evaluation of the proposed algorithm, two representative experiments are carried out. In the first one, a sensor is translated and rotated under motion acceleration and magnetic interference to test the performance of the algorithm quantitatively compared with other fusion methods. In the second one, a subject wears four SMU sensors on her lower limbs and carries out several motions recorded by a video camera. The tracking results by the proposed algorithm are compared with the recorded video through a quaternion-driven 3D human model animation. The SMU sensors used for the experiments are micro sensor chips ADIS16405 which are produced by the Analog Devices. Each SMU consists of a tri-axis accelerometer, a tri-axis gyroscope and a tri-axis magnetometer. The sampling frequency is $100 \mathrm{~Hz}$.

\subsection{Quantitative Comparison}

For quantitative comparison, three tests are carried out separately, during which the sensor's orientation is estimated by the proposed algorithm under motion acceleration and magnetic disturbance. FQA [3], Quaternion-based UKF [5], and directly integration from gyroscope data are also performed. In what follows, these three methods and our method are denoted as 'FQA', 'UKF', 'ING' and 'CKF' respectively. For the convenience of evaluation, quaternions from fusion methods are transformed into Euler angles.

The first test investigates the stability and accuracy of the filter when sensor signals are interfered by motion acceleration and magnetic disturbance. The sensor is placed statically most of the time; except from $8 \mathrm{~s}$ to $12 \mathrm{~s}$ and from $18 \mathrm{~s}$ to $21 \mathrm{~s}$, it is translated without rotation, during which motion acceleration is introduced. An iron cylinder is placed near the sensor twice, while one period is from $2 \mathrm{~s}$ to $6 \mathrm{~s}$ with the other from $15 \mathrm{~s}$ to $22 \mathrm{~s}$. Through all the process there is no rotation movements applied to the sensor. The true value of Euler angles about X, Y, and $\mathrm{Z}$ axes (roll, pitch and yaw) are zeros. The angles from these four methods are given in Fig.2. Given the ground truth of zeros, the estimation RMSE are calculated and summarized in Tab.1. 

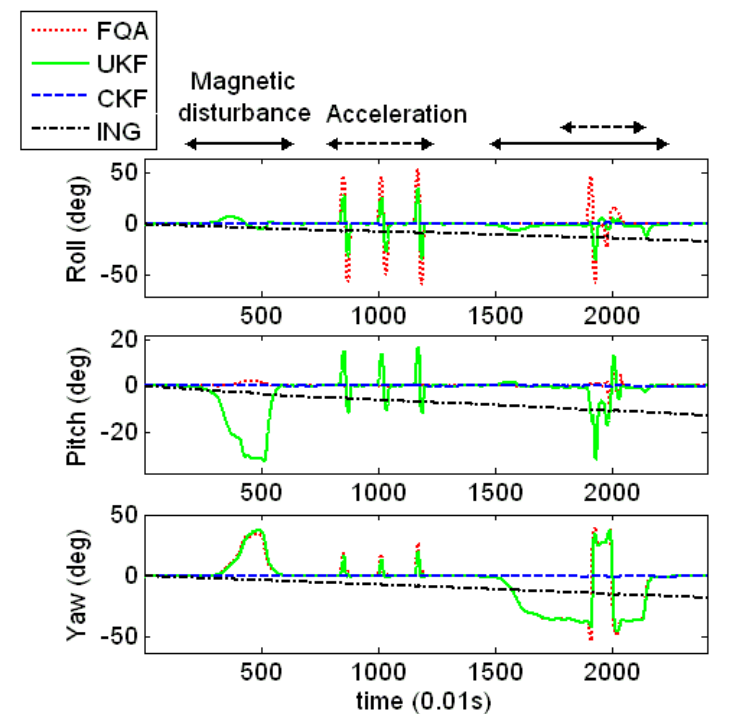

Fig.2 Euler angles (true value are zeros) where the sensor is static and translated. The solid and dashed arrows mark the periods of magnetic and translation acceleration interference respectively

Tab.1 RMSE under translation acceleration and magnetic disturbance

\begin{tabular}{|c|c|c|c|}
\hline Approach & Roll(deg) & Pitch(deg) & Yaw(deg) \\
\hline FQA & 10.6227 & 0.8794 & 18.2822 \\
\hline UKF & 5.9165 & 8.5585 & 18.0976 \\
\hline ING & 9.5822 & 7.6598 & 10.1693 \\
\hline CKF & 0.2904 & 0.1617 & 0.2838 \\
\hline
\end{tabular}

The second test investigates the stability of the filter under magnetic disturbance, when the sensor is rotated for - and $+90^{\circ}$ along $\mathrm{X}$ and $\mathrm{Y}$ axes respectively. When the rotation is about $X$ axis, the sensor is first rotated for $-90^{\circ}$ and then rotated back for $+90^{\circ}$ from $0 \mathrm{~s}$ to $6 \mathrm{~s}$, when there is no magnetic disturbance. From $7 \mathrm{~s}$ to $15 \mathrm{~s}$, the rotation is repeated, but the iron cylinder is placed near the sensor. The rotation about $\mathrm{Y}$ axis is almost the same as that of $\mathrm{X}$ axis, except that the magnetic disturbance is introduced from $8 \mathrm{~s}$ to $16 \mathrm{~s}$. The angles from these four methods are shown in Fig.3-4. Given the true value of zeros, the RMSE of pitch and yaw for rotation about $X$ axis, with those of roll and yaw for rotation about $\mathrm{Y}$ axis, are summarized in Tab.2 and Tab. 3 respectively.

Tab.2 RMSE of rotation about $X$ axis

\begin{tabular}{|c|c|c|}
\hline Approach & Pitch(deg) & Yaw(deg) \\
\hline FQA & 0.2920 & 19.6355 \\
\hline UKF & 0.5983 & 19.6820 \\
\hline ING & 5.1545 & 6.3202 \\
\hline CKF & 0.2414 & 0.4441 \\
\hline
\end{tabular}

Tab.3 RMSE of rotation about $\mathrm{Y}$ axis

\begin{tabular}{|c|c|c|}
\hline Approach & Roll(deg) & Yaw(deg) \\
\hline FQA & 0.3238 & 8.7164 \\
\hline UKF & 0.7492 & 8.6851 \\
\hline ING & 8.8193 & 5.7077 \\
\hline CKF & 0.2872 & 0.4660 \\
\hline
\end{tabular}
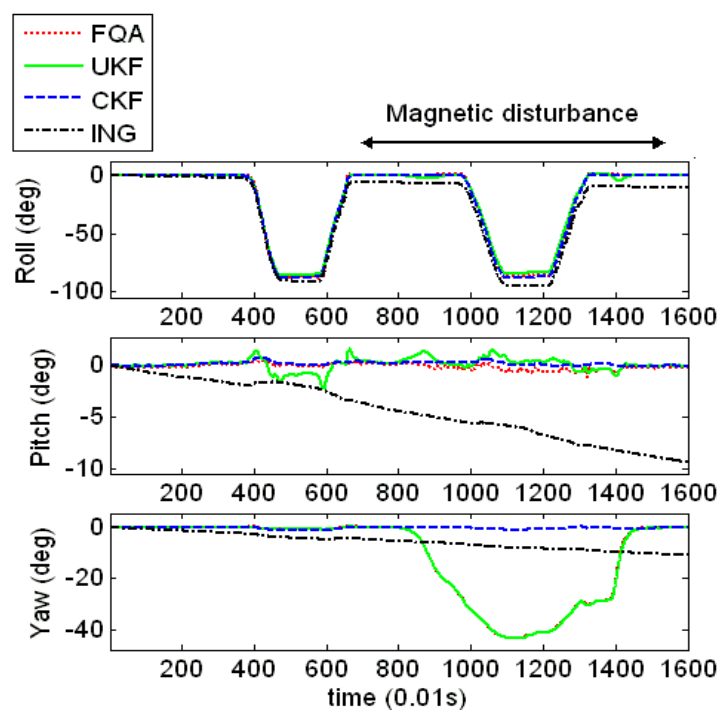

Fig.3 Euler angles (true value of pitch and yaw are zeros) where the sensor is rotated about $\mathrm{X}$ axis. The solid arrow marks the period of magnetic interference
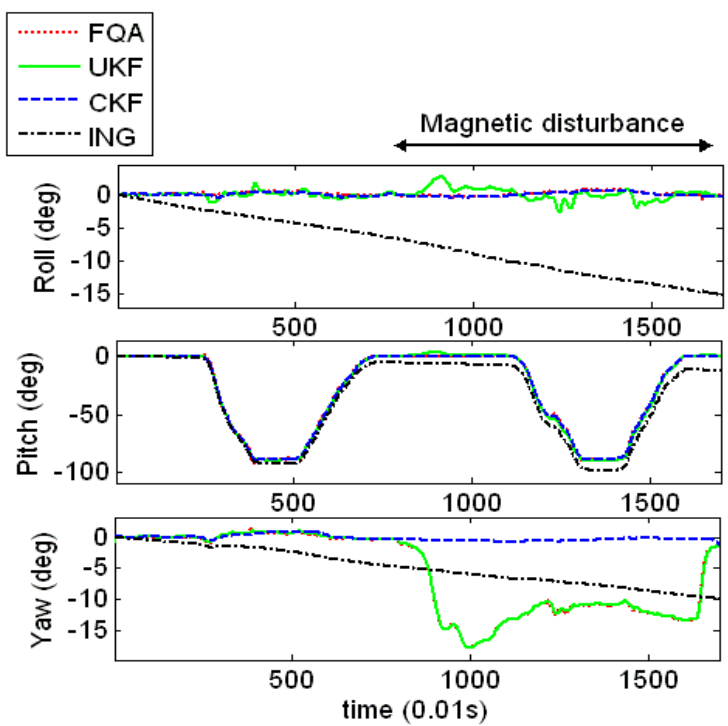

Fig.4 Euler angles (true value of roll and yaw are zeros) where the sensor is rotated about $\mathrm{Y}$ axis. The solid arrow marks the period of magnetic interference

The third test investigates the stability of the filter under motion acceleration, when the sensor is rotated for - and $+90^{\circ}$ along $\mathrm{Z}$ axis. The sensor is first placed statically with Z-axis pointing up vertically. Then it is rotated around $\mathrm{Z}$ axis for $-90^{\circ}$ and then rotated back for $+90^{\circ}$. During the rotation, translation with motion acceleration is performed at the same time. This process is repeated for several times with a total time length of 40s. The Euler angles from these four methods are shown in Fig.5, while local zoom results from time step 3501 to 3900 are shown in Fig.6. Given the ground truth of zeros, the estimation RMSE of roll and pitch are calculated and summarized in Tab.4.

From Fig.2-6, it can be seen that without correction from fusion of other sensing, Euler angles integrated directly from gyroscope signals become more and more 
inaccurate with the accumulation of time, whose error even exceeds more than 10 degrees after 40 seconds integration. This is the drift problem. FQA results fluctuate greatly for roll and pitch angles, and have peaks under motion acceleration; they fluctuate only for yaw under magnetic disturbance. UKF results fluctuate for all of the three angles under both motion acceleration and magnetic disturbance introduced.
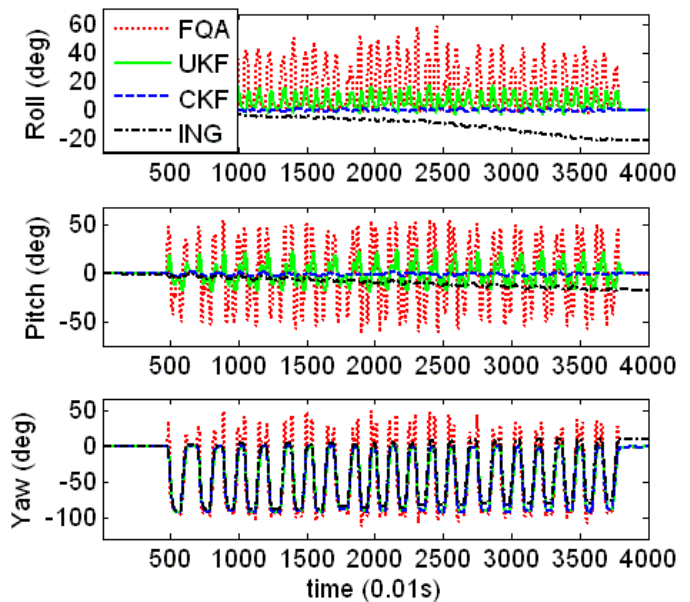

Fig.5 Euler angles (true value of roll and pitch are zeros), where the sensor is rotated about $\mathrm{Z}$ axis with translation acceleration
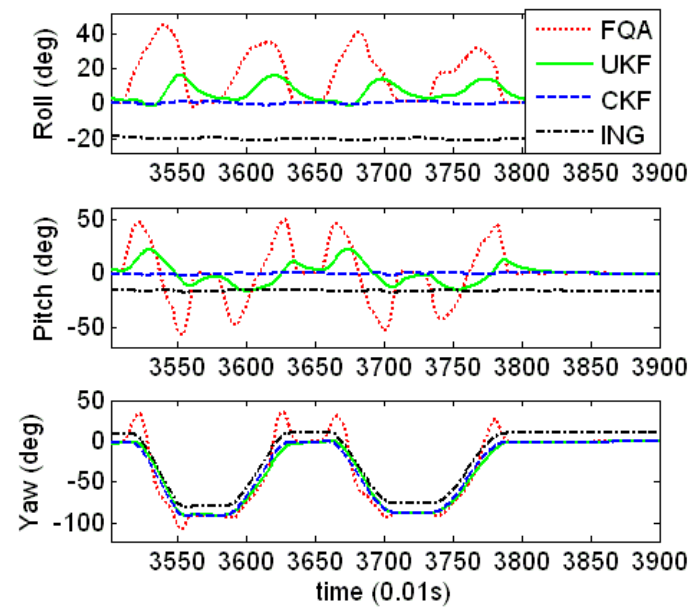

Fig.6 Euler angles (true value of roll and pitch are zeros) of local zoom results from 3501 to 3900 time steps

Tab.4 RMSE of each method

\begin{tabular}{|c|c|c|}
\hline Approach & Roll(deg) & Pitch(deg) \\
\hline FQA & 21.1547 & 25.7880 \\
\hline UKF & 7.1991 & 9.7208 \\
\hline ING & 10.8868 & 9.8013 \\
\hline CKF & 0.5627 & 1.1934 \\
\hline
\end{tabular}

From Tab.1-4 and Fig.2-6, it can be seen that the proposed CKF has the smallest RMSE among these four methods, and does not fluctuate much both under motion acceleration and magnetic disturbance. This is because during motion and magnetic interference, the confidence of the accelerometer and magnetometer signals decreases greatly, with the CKF putting less weight on them. The gyroscope signal plays a complementary effect on the fusion process. From the comparison of these results, our algorithm has shown its accuracy, stability and efficiency.

\subsection{Human Motion Capture}

In this experiment, the performance of the proposed algorithm is evaluated by extensive experiments: the lower limbs of a subject perform various types of actions and a video camera records these actions for comparison with our motion capture results. Four SMU sensors are used for the experiment, as we can see from Fig.7, two of them are placed on the sides of left and right thighs, and the other two are fixed on the front of each shank. Before the motion begins, an initialization process is performed to determine the relationship between the sensor frame and the body frame, which is not detailed in this paper. In the experiment, human motions performed by the subject are tracked by the proposed algorithm. The resulting orientation quaternions are visualized using 3D human model and animations $[7,8]$.

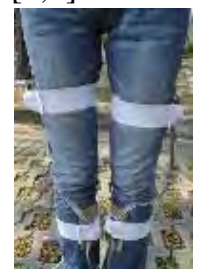

Fig.7 Sensor placement.

During the experiment, the subject first stands still to get the initial orientation, and then she does the following tasks: abduction and adduction of the thigh while the shank is relatively static with the thigh; flexion and extension of the thigh with the shank relatively static; abduction and adduction of the thigh as well as flexion and extension of the shank at the same time; flexion and extension of both the thigh and the shank. As we can see from Fig.8, the 3D human model animation driven by the fusion algorithm performs the exact same actions vividly and reliably compared to the real human motion. This verified the effectiveness of our algorithm.

\section{Conclusions and Future Work}

In order to overcome the drift problem in micro-sensor human motion capture, this paper presents a complementary Kalman filter (CKF), which is quaternionbased, to estimate drift-free orientation of body segments. Under the CKF framework, the integrated quaternion from gyroscope signals is corrected by the gravity and magnetic direction from accelerometer and magnetometer signals, respectively. The filter fuses sensor data of different sources adaptively based on their confidence, thus the interference from body acceleration and magnetic disturbance can be eliminated. Experimental results have demonstrated this property of the proposed algorithm; moreover, the 3D virtual human model driven by the fusion algorithm has shown stable and vivid animation of real human motion, and thus verified the effectiveness of the proposed algorithm. 


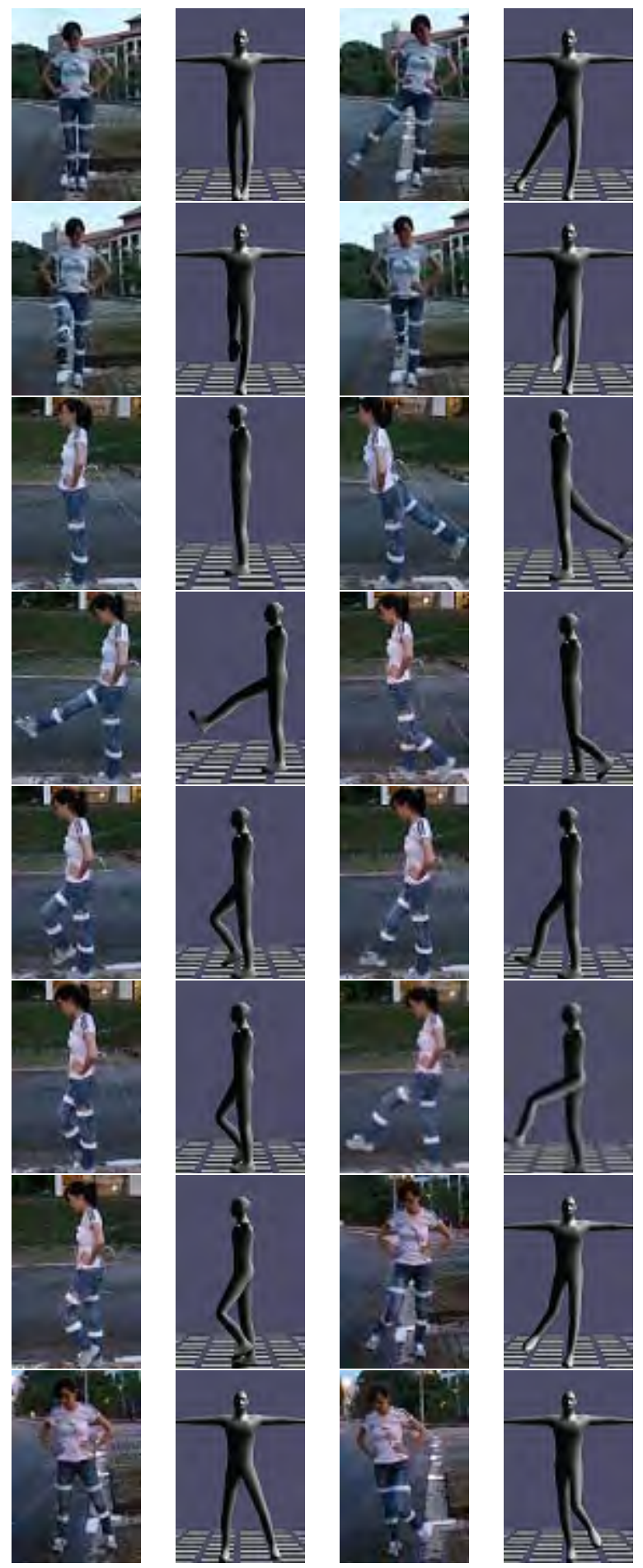

Fig.8 Lower limb motion capture

Our further work will be on more accurate sensor coordinate system calibration method, and comprehensive experiments to evaluate the performance and accuracy of the motion capture system.

\section{Acknowledgment}

The authors acknowledge Li Gang for his 3D human motion animation program, and Chen Jiang for his support on hardware platform. This paper is supported by the National Natural Science Foundation of China (Grant No.
60932001), and partially supported by CSIDM project 200802.

\section{References}

[1] Eric Foxlin, Inertial head-tracker fusion by a complementary separatebias Kalman filter, Virtual Reality Annual International Symposium 1996, Santa Clara CA, Mar. 30 -Apr. 3 1996, Proc. 1996, pp. 185-194, 267.

[2] Xiaoping Yun and Eric R. Bachmann, Design, Implementation, and Experimental Results of a Quaternion-Based Kalman Filter for Human Body Motion Tracking, IEEE Transactions on Robotics, Vol 22, No. 6, pp. 1216-1227, December 2006.

[3] Xiaoping Yun, Eric R. Bachmann, and Robert B. McGhee, A Simplified Quaternion-Based Algorithm for Orientation Estimation From Earth Gravity and Magnetic Field Measurements, IEEE Transactions on Instrumentation and Measurement, Vol 57, No. 3, pp. 638-650, March 2008.

[4] Daniel Roetenberg, Henk J. Luinge, Chris T. M. Baten, and Peter H. Veltink, Compensation of Magnetic Disturbances Improves Inertial and Magnetic Sensing of Human Body Segment Orientation, IEEE Transactions on Neural Systems and Rehabilitation Engineering, VOL 13, NO. 3, pp. 395-405, September 2005.

[5] Edgar Kraft, A quaternion-based unscented Kalman filter for orientation tracking, International Conference on Information Fusion 2003, Cairns, Australia, July 2003, Proc. 2003, pp. 47-54.

[6] D. Choukroun, I.Y. Bar-Itzhack and Y. Oshman, Novel quaternion Kalman filter, IEEE Transactions on Aerospace and Electronic Systems, Vol 42, No. 1. pp. 174-190, January 2006.

[7] Zhiqiang Zhang, Zhipei Huang and Jiankang Wu, Hierarchical information fusion for human upper limb motion capture, International Conference on Information Fusion 2009, Seattle, WA, USA, 6-9 July 2009, Proc. 2009, pp. 1704-1711.

[8] Gang Li, Zheng Wu, Xiaoli Meng, and Jiankang Wu, Modeling of Human Body for Animation by Microsensor Motion Capture, International Symposium on Knowledge Acquisition and Modeling 2009, Wuhan, China, Nov. 30 2009-Dec. 1 2009, Proc 2009, pp. 98-101. 